\title{
Effects on Quadriceps Muscle Strength Using EMG in Supervised vs Unsupervised Post-Operative Anterior Crucial Ligament Reconstruction
}

\author{
Sumit Saxena ${ }^{1}$, Aparna Sarkar ${ }^{2}$, A.K. Naik ${ }^{3}$ \\ ${ }^{1}$ PhD Scholar, Amity Institute of Physiotherapy, Amity University Uttar Pradesh, India, ${ }^{2}$ Professor Physiology, \\ Amity Institute of Physiotherapy, Amity University Uttar Pradesh, India, ${ }^{3}$ Professor, Department of Orthopedic, Dr. \\ RML Hospital, Delhi, India
}

\begin{abstract}
Anterior crucial ligament (ACL) injury is one of the most common ligament injuries in the knee. The ACL injury is the anterior ligament tear or sprain (ACL). The objective of this paper is to compare the effectiveness, recovery rate and the variance in the affected and non-affected limbs muscle strength/ activation potential readings of both knees in supervised v/s unsupervised groups of patients after undergone ACL reconstruction surgery at RML Hospital Delhi. The participants were divided into two groups (A-Supervised, and B-Unsupervised) through random sampling. The supervised exercise in group a performed according to the WILK protocol at RML hospital, and un-supervised group B performed the same WILK protocol at home. The method adopted for this study is to compare both Pre-operative and Post-Operative quadriceps(vastus medialis and vastus latralis) EMG readings of the respondents has been recorded using NEURON SPECTRUM 5 using NEUROSOFT Neuro-MEP.NET software at RML Hospital Delhi. The study findings indicated the significant difference in recovery rate as well as the difference in between supervised v/s unsupervised groups i.e. The average pre-operative-VM values of EMG- affected for supervised was $97.1 \mu \mathrm{V}$, and for unsupervised was $94.2 \mu \mathrm{V}$ and the average post-operative-VM values of EMG for supervised was $130.1 \mu \mathrm{V}(36 \%$ variance), and for unsupervised was $95.2 \mu \mathrm{V}$ (1\% variance). The average pre-operative-VM values of EMG- normal for supervised was $135.8 \mu \mathrm{V}$, and for unsupervised was $139 \mu \mathrm{V}$ and the average post-operative- $\mathrm{VM}$ values of EMG for supervised was $135.1 \mu \mathrm{V}$ (-7\% variance), and for unsupervised was $135.7 \mu \mathrm{V}(-6 \%$ variance $)$.
\end{abstract}

Keywords: Quadriceps; Muscle girth; ACL; EMG; Recovery.

\section{Introduction}

The anterior cruciate ligament (ACL) is one of the cruciate pairs in the human knee (the other being the posterior cruciate ligament). The two ligaments are also known as crossed ligaments, as they are arranged in a crossed formation. This name is appropriate as the ACL crosses the rear ligament to form an "X." This

\footnotetext{
Corresponding Author:

Sumit Saxena

PhD Scholar, Amity Institute of Physiotherapy, Amity

University Uttar Pradesh, India

saxena_sumit14@yahoo.co.in
}

consists of a heavy fibrous fibre which helps to regulate repetitive movement. This is done by restricting the joint's mobility. The anterior cruciate ligament is one of the four main ligaments of the knee, providing the anterior tibial displacement of $85 \%$ of the force of the restraint at 30 degrees and the knee flexion of 90 degrees ${ }^{1-5}$. The quadriceps is critical for lowering/reducing limb control during dynamic activity and quadriceps weakness can change movement strategies, which can lead to injury. To prepare patients optimally to return to full activity it is necessary to restore full quadriceps function. Before the severity of quadriceps deficits can be effectively counteracted, however, there must be a deep understanding as to why the weakness of the quadriceps persists throughout recovery. Quadriceps disuse atrophic 
arises after the immobilization of the knee joint and may lead to the weakness of the quadriceps after ACL injury and after reconstruction. Deficits of 3\% are 12-18 months post-operative, involume 6 and cross-sectional area (CSA). Similar amounts of quadriceps atrophy were documented, although no link between atrophy and force was found. The authors concluded that the weakness of quadriceps alone did not lead to incomplete volitional muscle activation ${ }^{6,7}$.

Atherogenic inhibition of muscles results from an activation of several different joint receptors that function upon the synapses of the inhibitory interneurons on the joint muscle motor neuron pool. Cryotherapy has more "disinhibition" or activation of the VM than the transcutaneous neuromuscular electrical stimulation community of longer-life effects ${ }^{1,12-14}$. Disinhibition is defined as "a return of some measure of recruitment to baseline or preinjury levels."

\section{Materials and Method}

80 patients were selected for participants who volunteered to participate in recent knee surgery. Those who have undergone surgery post operation after 3 weeks of data collection can take part.; one was omitted following secondary screening and the inclusion criteria alone were not fulfilled. Another person reported for MRI but did not report the CAR test. 80 patients were subjected tosemitendinosus graft with the inside out technique of ACL reconstruction of CAL (62 males, 18 females). Medical clarification for the restoration of full activity before reconstruction was given to patients, reported pre-operation screening and assessment ACL reconstruction) ${ }^{2}$.Exclusion criteria as following.

(1) Any lower extremity injury except recent ACL;

(2) partial or full meniscectomy;

(3) cumulative ligament damage to other ligaments ACL injury; or

(4) Common heart conditions. Even pregnant women were excluded.

The volunteer respondents enrolled focused on physical therapy to facilitate postoperative knee rehabilitation through pre-established rehabilitation protocols (WILK). The subjects were seated on a padded table with both knees firmly supported against a wall. At the beginning of the session, the knee girth measurements were carried out at the upper margin of the patella. According to a 10-point visual analogue scale, the pain levels were reported explicitly to measure subjective symptoms during knee operations. At the beginning of each data collection session, a review period was conducted for all subjects. This time the subjects were familiarized with the research procedure and practised the full $3,12,15,16$. Joint effusion was measured by lying supine on couch from superior pole of the patella in patients. A tag placed $1 \mathrm{~cm}$ near the top pole, and a tape scale was used to measure the circumference of the knee. An average of three MVIC knee extension periods with a minimum of 2 minutes ' rest between repeating until no torque change was detected by the investigator. and the QAF evaluation was used as a benchmark ${ }^{4,17,18}$. The eligible patients underwent anterior reconstruction in cross-ligaments (ACL R) using inside out technique;

Procedure: Enrolled volunteers have registered for the study has to undergo measurement of MVIC 2 weeks Pre Operation then 12 to 14 weeks post-operation. Knee rehabilitation with pre-established recovery guidelines for outpatient physical therapy was started. The subjects were seated on a padded table with their backs against a wall completely extended on both legs. At the beginning of each data collection session, a review period was conduct. Maximum voluntary isometric contraction (MVIC) was calculated with the help of the EMG machine for all subjects. This time, the participants were familiarised with the study procedure and the overall MVIC (three to five experimental studies) performed on both the limbs normal and surgical. During three QS trials, EMG measurements of the VM, and VL from the surgical leg were obtained (Figure 1). The subjects were given verbal commands to press the back of their surgical knees as hard as possible into the padded table below their knees before they were ordered to stop. QS Test lasted 5 seconds with a rest of 30 seconds between each test. The subjects were advised to relax as the data was processed. The participants were asked to assess their pain after the three QS tests with a 10 point visual analogue scale using the verbal commands "up," "push" and "stop," and were instructed to stretch their knees with full effort for $5 \mathrm{~s}$, with $30 \mathrm{~s}$ remaining between two trials. Knee extensor MVIC EMG activity was obtained with the participant lying supine on an examination bed with a foam roller (diameter $15 \mathrm{~cm}$ ) placed below the knee to ensure a slight knee flexion. The pelvis was fixated to the examination bed by a non-elastic strap. The hand was placed on top of the lower leg in a distance corresponding to the width of two fingers above the medial malleolus. This procedure has been recorded with 
excellent intra-class correlation coefficient $(0.929,95$ percent confidence interval $[\mathrm{CI}]=0.857-0.966)$. 36 All MVIC's lasted 4 secs to allow optimum muscle activity and good verbal encouragement. Three MVIC tests with a 30-second rest between each test were conducted to avoid fatigue build-up for each muscle.

Assessment: Detail assessment was performed according to the assessment Performa measuring the mid-thigh circumference and special tests and strength of the musclePre-operation, andpost-operation 12 to 14 weeks. WILK protocol was adopted for the strengthening exercise for both the groups for 3 months post-operative.

Quadriceps EMG recording and data analysis: NEURON SPECTRUM 5 using NEUROSOFT NeuroMEP.NET software surface measurements are carried out. Single differential bar-electrode Ag-AgCl (DE-2.1; Delsys Inc.) were fastened to the medial malleolus of the non-chirurgical body using an electrolyte gel and an adhesive skin interface over a set inter-electrode length of $1 \mathrm{~cm} \mathrm{27.EMG} \mathrm{data} \mathrm{are} \mathrm{obtained} \mathrm{for} \mathrm{the} \mathrm{following}$ muscles of the surgical leg: VM (two finger-widths near the superomedial border of the patella along the expected fibre line), and vast lateralis (VL, four fingerwidths next to the superolateral border of the patella). Bandpass filters $(20-500 \mathrm{~Hz})$ and notch filters $(60 \mathrm{~Hz})$ were used. During the maximum voluntary isometric contraction (MVIC) the EMG signal was obtained for $5 \mathrm{~s}$ for the monitoring of quadriceps and the knee was extended (isometric effects of the monitored muscles). For each of the muscles, 5-s MVIC root-mean-square (RMS) was tested for three tests in the middle of three seconds. The highest value was then evaluated in the three consecutive trials.

Statistical tests: The SPSS 24 (Statistics System for Social Science) was used for statistical analysis. The paired t-test and was performed, which was having a significance $p$-value (less than 0.05$)^{19-21}$.

\section{Results}

Figure 1 and figure 2 showed the data for supervised and Un-supervised groups. The following information was tabulated in the Excel spreadsheets: sex, age, PreOperative EMG VL, Pre-Operative EMG VM, PostOperative EMG VL, and Post-Operative EMG VM for both supervised and unsupervised groups. For affected and Normal leg readings pre and post-operation for both supervised and unsupervised groups. A total of (80) participants were divided into two groups. The findings of the data analysed is mentioned group wise below:

- The average pre-operative-VL values of EMGaffected for supervised was $95.2 \mu \mathrm{V}$ and for unsupervised was $94.6 \mu$ Vand the average postoperative-VL values of EMG for supervised was $126.9 \mu \mathrm{V}(32 \%$ variance), and for unsupervised was $135.7 \mu \mathrm{V}$ (43\% variance).

- The average pre-operative-VL values of EMGnormal for supervised was $144.5 \mu \mathrm{V}$, and for unsupervised was $145 \mu \mathrm{V}$ and the average postoperative-VL values of EMG for supervised was $133.8 \mu \mathrm{V}$ (-7\% variance), and for unsupervised was $121.4 \mu \mathrm{V}(-16 \%$ variance).

- Similarly, the average pre-operative-VM values of EMG- affected for supervised was $97.1 \mu \mathrm{V}$, and for unsupervised was $94.2 \mu \mathrm{V}$ and the average postoperative-VM values of EMG for supervised was $130.1 \mu \mathrm{V}(36 \%$ variance), and for unsupervised was $95.2 \mu \mathrm{V}$ (1\% variance).

- The average pre-operative-VM values of EMGnormal for supervised was $135.8 \mu \mathrm{V}$, and for unsupervised was $139 \mu \mathrm{V}$ and the average postoperative-VM values of EMG for supervised was $135.1 \mu \mathrm{V}$ (-7\% variance), and for unsupervised was $135.7 \mu \mathrm{V}(-6 \%$ variance) figure 1 and figure 2 . 


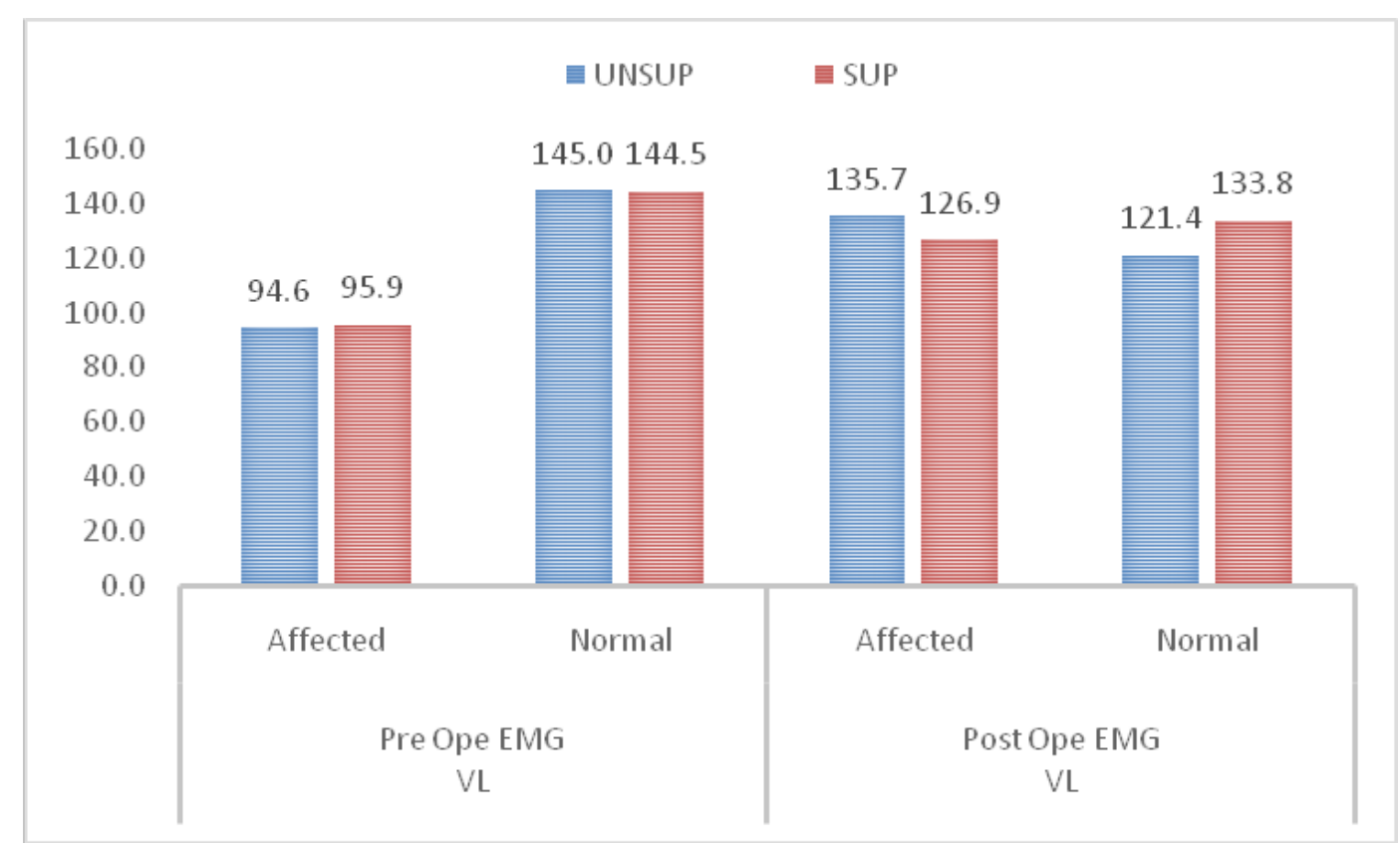

Figure 1: EMG values for VL for supervised and unsupervised groups of patients

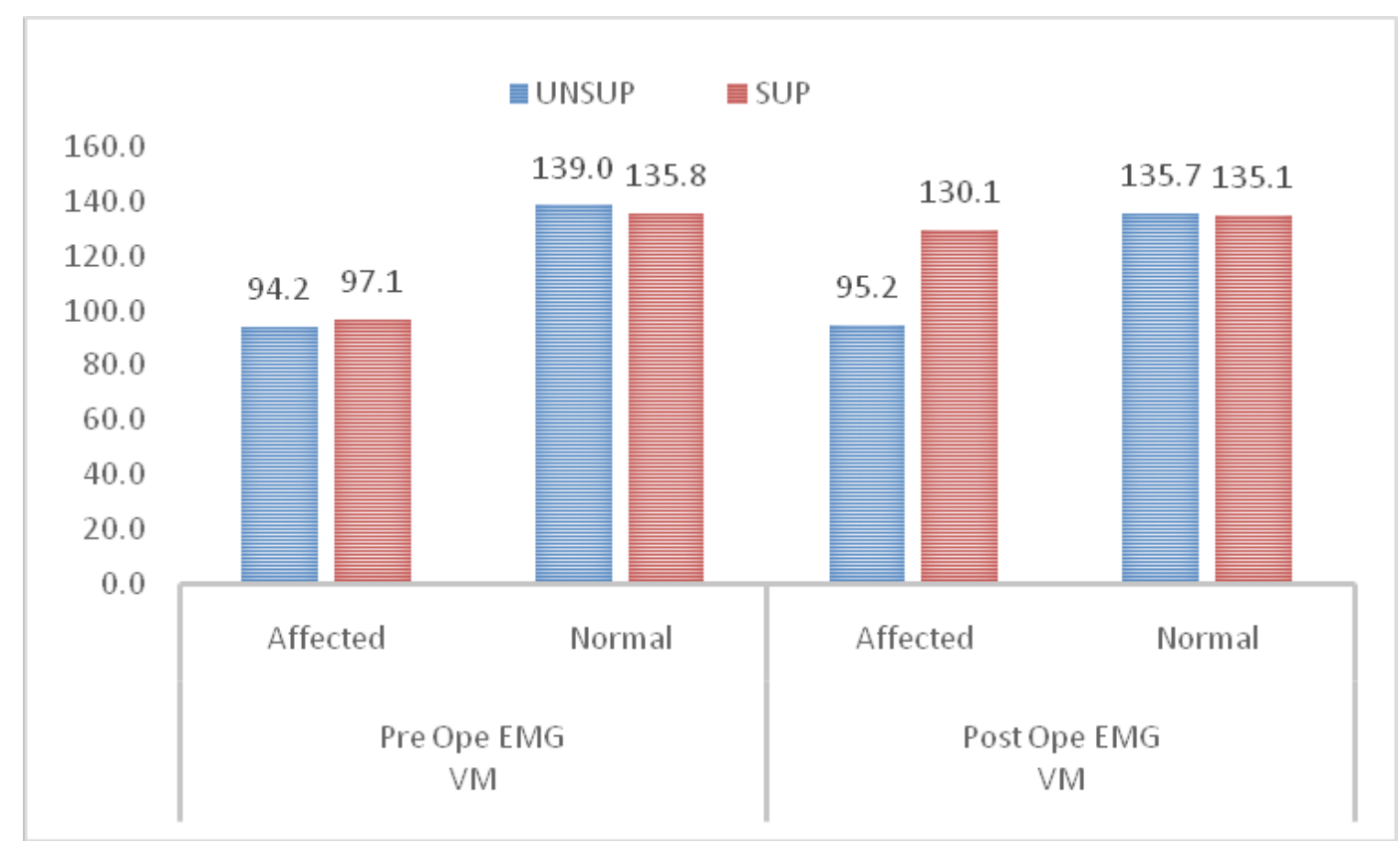

Figure 2: EMG values for VM for supervised and unsupervised groups of patients

\section{Discussion}

Our hypothesis that supervised exercise have a better impact on strengthening and post-operative recovery in comparison with the unsupervised exercises knee surgery leads to an improvement in EMG quadriceps muscles amplitude. We also noted the VM was the muscle in which EMG after surgery that could vary because of atherogenic inhibition of the muscle ${ }^{1,11,13,18,22-24}$. Although traditional views and clinical observation suggest that $\mathrm{VM}$ is more inhibited after joint injury than other Quadriceps heads, recent findings suggest uniform weakness, atrophy and quadriceps deactivation following joint injury.

The study also showed that due to the unequal strength of both limbs, weight-bearing asymmetry in both limbs would also cause difficulties in dynamic operation. This research also gave us a quantitative meaning in relation to the difference in strength recovered in both groups, 
which are also beneficial in the future for the individual to tailor the rehab protocol. The intensity of exercise, as determined in a given percentage of maximum muscle contraction strength, is a fundamental element in muscle strength progression. Surface electromyography (EMG) is also used for indicating exercise intensity as a positive linear association between isometric muscle strength and amplitude was previously reported. In addition, during dynamic muscle contraction, there are positive proportionate relationships between muscle strength and EMG amplitude, although this relationship can be slightly curviliner in some muscles. Normalizing the EMG amplitude for the average EMG amplitude obtained under isometric conditions improves measurement reliability. Therefore, the standardized EMG (nEMG) amplitude measured as percent of the mean EMG amplitude provides an indicator of exercise strength and is widely used in exercise evaluation studies. Nonetheless, traditional strength training method are still not used in clinical practice and for home-based recovery. One of the key areas of focus in recovery following ACL reconstruction is to implement safe exercises which target quadriceps muscles gradually at appropriate levels of activity. However, reduced lower limb muscle strength is documented, often far beyond post-operative rehabilitation, not only in the quadriceps but also in the hamstring muscles after ACL injury/ reconstruction. During strong dynamic movements, hamstrings co-activation is critical for dynamic knee joint stabilisation and excessive prevention.

\section{Conclusion}

With the above study, the authors concluded that supervised exercises in post-operative ACL reconstruction plays a very significant role in the recovery and also reduces the chances of post-operative complications remarkably.

Ethical Clearance: This research was approved by the Ethical committee of Amity University Noida and Dr RML Hospital Delhi. Before participation, all subjects signed informed consent documents and the interests of all subjects are secured.

Source of Funding: Self funded.

Conflict of Interest: Nil.

\section{References}

1. Sinha S, Naik AK, Maheshwari M, Sandanshiv
S, Meena D. Anterior Cruciate Ligament Reconstruction with Tibial Attachment Preserving Hamstring Graft without Implant on Tibial Side. 2018;52(2):170-176. doi:10.4103/ortho.IJOrtho

2. Beutler AI, Cooper LW, Kirkendall DT, Garrett WE. Closed Chain Exercises : Implications for Ligament Reconstruction. 2002;37(1):13-18.

3. Zebis MK, Sørensen MH, Lauridsen HB, et al. Electromyography Evaluation of Bodyweight Exercise Progression in a Validated Anterior Cruciate Ligament Injury Rehabilitation Program. 2019;98(11):998-1004. doi:10.1097/ PHM.0000000000001232

4. Loro WA, Thelen MD, Rosenthal MD, Stoneman PD, Ross MD. Or thopaedic Surger y The effects of cryotherapy on quadriceps electromyographic activity and isometric strength in patient in the early phases following knee surgery. 2019;27(1):18. doi:10.1177/2309499019831454

5. Colby S, Francisco A, Yu B, Kirkendall D, Finch M, Garrett W. The American Journal of Sports Medicine Electromyographic and Kinematic Analysis of Cutting Maneuvers Implications for Anterior Cruciate Ligament Injury. The American Journal of Sports Medicine. 2000;28(2):234-240. doi:10.1016/S1440-2440(99)80066-8

6. de Vries AJ, van den Akker-Scheek I, Haak SL, Diercks RL, van der Worp H, Zwerver J. Effect of a patellar strap on the joint position sense of the symptomatic knee in athletes with patellar tendinopathy. Journal of Science and Medicine in Sport. 2017;20(11):986-991. doi:10.1016/j. jsams.2017.04.020

7. Click AB. Taking something that is not your right: Egyptian students' perceptions of academic integrity. Libri. 2014;64(2):109-123. doi:10.1515/ libri-2014-0009

8. Pandy MG, Shelburne KB. Dependence of cruciateligament loading on muscle forces and external load. Journal of Biomechanics. 1997;30(10):10151024. doi:10.1016/S0021-9290(97)00070-5

9. Plastaras CT, Rittenberg JD, Rittenberg KE, Press J, Akuthota V. Comprehensive functional evaluation of the injured runner. Physical Medicine and Rehabilitation Clinics of North America. 2005;16(3):623-649. doi:10.1016/j. pmr.2005.02.005

10. Code CV. Note: Page numbers followed by. 
1964:75-77. doi:10.1016/B978-0-12-8041345.09993-1

11. Ciccotti MG, Kerlan RK, Perry J, Pink M. An Electromyographic Analysis of the Knee During Functional Activities 2: the Anterior Cruciate Ligament-Deficient and LigamentReconstructed Profiles. American Journal of Sports Medicine. 1994;22(5):651-658. doi:10.1177/036354659402200513

12. Biscarini A, Contemori S, Busti D, Botti FM, Pettorossi VE. Knee fl exion with quadriceps cocontraction: A new therapeutic exercise for the early stage of ACL rehabilitation. 2016;49:38553860. doi:10.1016/j.jbiomech.2016.10.026

13. Sinha S, Naik AK, Meena D. Creation of femoral tunnel by outside-in technique for ACL reconstruction : an analysis. 2014:1709-1716. doi:10.1007/s00402-014-2078-5

14. No Title. 2019. doi:10.5152/EurJTher.2019.19039

15. Engineering R. Rehabilitation Engineering and Assistive Technology Call for paper General Chair : The 2 nd Shanghai International Symposium on Human-Centered Robotics Call for Paper General Chair : 2018;(July):14-15.

16. Thomas AC, Wojtys EM, Brandon C, Palmierismith RM. Journal of Science and Medicine in Sport Muscle atrophy contributes to quadriceps weakness after anterior cruciate ligament reconstruction. Journal of Science and Medicine in Sport. 2016;19(1):7-11. doi:10.1016/j.jsams.2014.12.009

17. Verma NN, Ahmad CS, Cole BJ, Jr BRB. Reconstruction. Arthroscopy: The Journal of ArthroscopicandRelated Surgery.2016;32(8):16121621. doi:10.1016/j.arthro.2016.01.033

18. Sinha S, Jain VK, Upadhyay G, Delhi N. Trans-tibial guide wire placement for femoral tunnel in single bundle anterior cruciate ligament reconstruction. 2015;49(3):352-356. doi:10.4103/00195413.156224

19. Dixit S, Mandal SN, Thanikal J V, Saurabh K. Evolution of studies in construction productivity: A systematic literature review (2006-2017). Ain Shams Engineering Journal. 2019. doi:10.1016/j. asej.2018.10.010

20. Dixit S, Sharma K. An Empirical Study of Major Factors Affecting Productivity of Construction Projects. In: Babu KG, Rao HS, Amarnath Y, eds. Emerging Trends in Civil Engineering. Singapore: Springer Singapore; 2020:121129.21. Dixit S, Sharma K, Singh S. Identifying and Analysing Key Factors Associated with Risks in Construction Projects. In: Babu KG, Rao HS, Amarnath Y, eds. Emerging Trends in Civil Engineering. Singapore: Springer Singapore; 2020:25-32.

22. Wilson WK, Morris R, Coskey A, Smith B, Gugala Z. Quadriceps augmentation of undersized hamstrings during ACL reconstruction. Knee. 2019;26(1):73-78. doi:10.1016/j.knee.2018.11.012

23. Rhatomy S, Hartoko L, Setyawan R, et al. Journal of Clinical Orthopaedics and Trauma Single bundle ACL reconstruction with peroneus longus tendon graft : Journal of Clinical Orthopaedics and Trauma. 2019;(xxxx):6-10. doi:10.1016/j.jcot.2019.09.004

24. Adeoye O, Christianah O, Spirina G, Cui D, Lynch JC, Lehman MN. ISMS2017-1-001 Radiofrequency electromagnetic radiation from cell phone causes defective testicular function in male ISMS20171-003 Comparative study of the ear of black and white ISMS2017-1-002 Influence of variations of coronary artery branches on blood. Annals of Anatomy - Anatomischer Anzeiger. 2017;1:6-334. doi:10.1016/j.aanat.2017.05.003 